\title{
A rare case of macroprolactinoma in a patient with Mayer-Rokitansky-Kuster-Hauser (MRKH) syndrome
}

\section{Nur Aisyah Zainordin, Fatimah Zaherah Mohd Shah, Nur Aini Eddy Warman, Sharifah Faradila Wan Muhammad Hatta, Aimi Fadilah Mohamad and Rohana Abdul Ghani}

Internal Medicine Discipline, Faculty of Medicine, University Technology MARA, Jalan Hospital, Sungai Buloh, Selangor, Malaysia
Correspondence should be addressed to N A Zainordin

Email

aisyahzack@gmail.com

\section{Summary}

A 17-year-old lady presented with primary amenorrhoea, headache, nausea and lethargy. She had delayed pubertal development that also includes under-developed breast (Tanner Stage 2). Hormonal investigations showed a high serum prolactin level of $1680000 \mathrm{mIU} / \mathrm{L}$ (normal value: 45-375 mIU/L), with low oestradiol, progesterone, follicular-stimulating hormone and luteinizing hormone. Early morning cortisol level was $206 \mathrm{nmol} / \mathrm{L}$ (normal value: $>450 \mathrm{nmol} / \mathrm{L}$ ), thyroxine was $7.5 \mathrm{pmol} / \mathrm{L}$ (normal value: $9.0-24.0 \mathrm{pmol} / \mathrm{L}$ ) with TSH $5.091 \mathrm{mIU} / \mathrm{L}$ (normal value: 0.4-4.5 mlU/L). A pituitary MRI showed a $2.7(\mathrm{AP}) \times 3.7(\mathrm{~W}) \times 4.6 \mathrm{~cm}(\mathrm{CC})$ macroadenoma, with invasion into the left cavernous sinus and encasement of cavernous portion of the left internal carotid artery. MRI pelvis showed absent uterus, cervix and 2/3 upper vagina confirming Mullerian hypoplasia. Cytogenetics showed 46XX. These findings were suggestive of Mayer-Rokitansky-Kauser-Hauser (MRKH) syndrome with the presence of a pituitary macroprolactinoma and panhypopituitarism. She was treated with hydrocortisone, levothyroxine and cabergoline. Repeated MRI showed a reduction in tumour size by approximately $50 \%$. This case illustrated a rare coexistence of these two conditions, being only the third reported case in the world. In addition, this would be the first case of a functioning pituitary adenoma in a patient with MRKH syndrome.

\section{Learning points:}

- Comprehensive hormonal and radiological investigations are important in the management of a young patient with primary amenorrhoea.

- Coexistence pathology of two separate pathologies should be considered in patient presenting with primary amenorrhoea.

- Early diagnosis of MRKH or any disorders of sex development should be treated early, providing pharmacological, surgical, psychological and emotional support to the patient and reducing risk of associated complications.

- Abnormal pituitary hormones, particularly panhypopituitarism, would impose greater impact not only psychologically but also metabolically leading to cardiovascular, morbidity and mortality risks in this patient if not treated early.

- A multidisciplinary approach is necessary for patients presenting with MRKH to ensure appropriate treatments and follow-up across the lifespan of the patient. 


\section{Background}

Mayer-Rokitansky-Kuster-Hauser (MRKH) syndrome or Mullerian agenesis is a congenital abnormality, characterised by failure of the Mullerian duct to develop, resulting in complete or partial absence of the cervix, uterus and vagina. The condition is associated with normal development of secondary sexual characteristics and normal 46XX karyotype. It is caused by an interruption in the development of the Müllerian duct system, occurring during the fifth and sixth weeks of gestation (1). MRKH syndrome is further classified as type I (isolated uterovaginal aplasia) and type II (associated with extragenital manifestations), of which the latter typically includes renal, vertebral, auditory and cardiac defects malformation (2). The incidence of this anomaly is estimated to range from 1:4000 to 1:5000 (3). Prolactinsecreting tumour or prolactinoma is the most common secretory tumours of the pituitary gland accounting for up to $40 \%$ of total pituitary adenomas (4). Prolactinoma has been reported in patients between age 2 and 80 years, occurring most frequently in women from the second to the fifth decades of life (5). Prolactinomas are even rarer in children and adolescents. But they represent 50\% of all pituitary adenomas, which accounts for $2 \%$ of all intracranial tumours in the age group (5).

However, they are rarely accompanied by congenital anomalies. This report highlights not only a rare clinical presentation but this would be the first case of a prolactinsecreting pituitary macroadenoma associated with MRKH syndrome. It is currently unclear if there is a hormonal or metabolic explanation to this coexistence.

\section{Case presentation}

A 17-year-old lady presented with primary amenorrhoea, associated with occasional headache, nausea and lethargy, which had been worsening in duration and intensity over the last 2 years. She denied any abnormalities in her vision including visual field. She had no other medical illnesses and had not been taking any medications or supplements. She had four other siblings, of which two of her sisters had normal menarche at ages below 15 years old. Additionally, there was no history of congenital anomalies among her family members. Her antenatal history was unremarkable with full-term vaginal delivery. There was no significant maternal obstetrical history, particularly no exposure to hormonal therapy or radiation. She did not smoke and attained moderate academic achievement at school.
On examination, her height was $140 \mathrm{~cm}$, weight was $65.9 \mathrm{~kg}$ and BMI was $33 \mathrm{~kg} / \mathrm{m}^{2}$. During her initial presentation, she was hypotensive with blood pressure of $85 / 69 \mathrm{mmHg}$, which subsequently normalised after initiation of treatment. She demonstrated incomplete pubertal development which includes breast of Tanner 2, pubic hair Tanner 3 axillary hair was sparse, Tanner 1 and features of normal external genitalia. Her abdomen was soft and non-tender. Her visual field examinations showed a left temporal visual defect and a normal right visual field.

\section{Investigation}

The preliminary hormonal profiles are listed in Table 1 . There was evidence of a remarkably high serum prolactin level, associated with low level of follicular-stimulating hormone, luteinizing hormone, oestradiol, progesterone and testosterone. Her FT4 was low with normal level of TSH. Despite low value of cortisol, dynamic testing was not carried out as patient showed signs of hypocortisolism including hypotension. Her blood pressure was normalised after hydrocortisone was started.

As part of the initial investigation for primary amenorrhoea, transabdominal ultrasonography was performed which was unable to visualize the uterus and ovaries. Subsequently, MRI of the pelvis confirmed the absence of uterus, cervix and $2 / 3$ of the upper vagina. Refer Fig. 1. Both ovaries appeared small with tiny cyst. A consequent MRI of the pituitary showed an enlarged pituitary gland occupying the pituitary fossa with suprasellar extension measuring 2.7 (anterior-posterior, AP) $\times 3.7$ (Width, W) $\times 4.6 \mathrm{~cm}$ (craniocaudal, CC). The macroadenoma was hypointense in the $\mathrm{T} 1 \mathrm{~W}$ and mixed iso- and hyperintense on $\mathrm{T} 2 \mathrm{~W}$ sequence. It enhanced heterogeneously in contrast (Fig. 2).

Table 1 Result of the patient's hormonal profile.

\begin{tabular}{|c|c|c|}
\hline & Level & Normal range \\
\hline Prolactin & $168000 \mathrm{mIU} / \mathrm{L}$ & 45-375 mIU/L \\
\hline $\mathrm{FSH}$ & $2.38 \mathrm{U} / \mathrm{L}$ & 1.4-18.1 U/L \\
\hline LH & $0.52 \mathrm{U} / \mathrm{L}$ & $1.5-9.3 \mathrm{U} / \mathrm{L}$ \\
\hline Progesterone & $<0.3 \mathrm{nmol} / \mathrm{L}$ & $0.5-1.0 \mathrm{nmol} / \mathrm{L}$ \\
\hline Oestradiol & $<37 \mathrm{pmol} / \mathrm{L}$ & 72-529 pmol/L \\
\hline Testosterone & $<0.45 \mathrm{nmol} / \mathrm{L}$ & $3.0-27.35 \mathrm{nmol} / \mathrm{L}$ \\
\hline TSH & $4.83 \mathrm{mIU} / \mathrm{L}$ & 0.55-4.78 mIU/L \\
\hline fT4 & $8.6 \mathrm{pmol} / \mathrm{L}$ & 11.5-22.7 pmol/L \\
\hline
\end{tabular}

$\mathrm{FSH}$, follicle-stimulating hormone; $\mathrm{LH}$, luteinizing hormone; TSH, thyroid-stimulating hormone. 


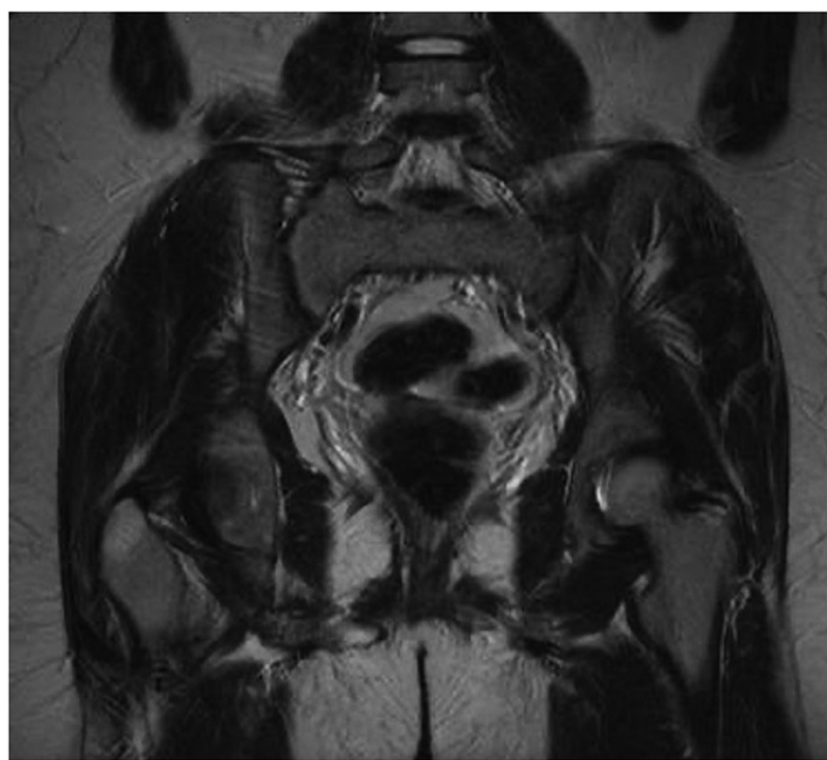

Figure 1

MRI pelvis coronal view showed absent uterus, cervix and 2/3 upper vagina confirming Mullerian hypoplasia.

Her genetic study showed normal karyotyping of $46 \mathrm{XX}$. Based on the findings, a diagnosis of type 1 Mayer-Rokitansky-Kauser-Hauser syndrome was made, with the presence of a macroprolactinoma and panhypopituitarism.



Figure 2

MRI of pituitary in coronal view T1 sequence showed $2.7(A P) \times 3.7(W) \times$ $4.6 \mathrm{~cm}$ (CC) pituitary macroadenoma, with mass effect, infiltration into left cavernous sinus and encasement of cavernous portion of left Internal Carotid Artery (ICA.

\section{Treatment}

Shewas promptlytreated with hydrocortisoneaccompanied by levothyroxine and cabergoline. Surgical option has been discussed with her in view of large pituitary tumour. However, in view of the diagnosis of macroprolactinoma, she was opted for medical therapy of cabergoline. Her dose was adjusted from 0.5 to $1.5 \mathrm{mg}$ per week according to her prolactin level response to the cabergoline dose. She was also provided counselling regarding future partner and off-spring.

\section{Outcome and follow-up}

She underwent a repeat MRI of the pituitary after 6 months of treatment which showed a significant reduction in the tumour size of $1.3 \times 1.7 \times 1.6 \mathrm{~cm}$. A repeat pelvic MRI was planned to look for any uterine development following oestrogen treatment but has been postponed due to COVID19 pandemic.

She remained well and continued on the medical follow-up at the institution.

\section{Discussion}

The diagnosis of MRKH syndrome was evident due to the absence of uterus and vagina on pelvic MRI and ultrasonographic examination, in the presence of a 46,XX karyotype. However, the presence of hypogonadotropic hypogonadism was an inconsistent feature for MRKH. This was subsequently detected to be due to a large macroprolactinoma causing panhypopituitarism, confirmed by the abnormal hormonal profiles and MRI pituitary findings. Patients with MRKH syndrome usually present with both normal stature and secondary sexual developments (3). Therefore, to the best of our knowledge, this report would be the first case of hypogonadotropic hypogonadism in $\mathrm{MRKH}$, secondary to a large pituitary macroadenoma.

MRKH syndrome has been reported in approximately $16 \%$ of patients with primary amenorrhoea, thus considered as the second most common cause of primary amenorrhoea after ovarian failure (3). The reported prevalence of the condition ranges between 1:4000 and 1:5000 females (6). The aetiology of MRKH syndrome is unknown, mainly sporadic, although familial cases have been reported to suggest some degree of inheritance in certain families (3). In MRKH syndrome, both ovaries are typically normal in appearance as well as function. Ovary anomalies are rare and are only found in approximately 
$5-10 \%$ of cases (7). Endocrine hormone disturbance previously has not reported FSH, LH, androgens and oestradiol, which are generally considered to be normal in MRKH syndrome (7).

MRKH syndrome is generally classified into two types, with type I being seen in isolated cases of utero-vaginal agenesis, while the more common type II is seen in cases of utero-vaginal agenesis associated with extra-genital anomalies, including renal, skeletal, auditory and cardiac anomalies (7). Among the patients with associated extragenital malformations, renal anomalies were the most common. As the patient reported here showed no renal, cardiac or skeletal malformation, she was diagnosed as having MRKH type 1.

The differential diagnoses of her condition included androgen insensitivity, transverse vaginal septum and imperforated hymen. Androgen insensitivity syndrome is distinguished from MRKH syndrome by decreased pubic and axillary hair, a 46,XY karyotype, elevated testosterone level and presence of rudimentary testicles on magnetic resonance images (6) which were excluded in this patient. The latter 2 differential diagnoses were also easily excluded from the imaging results.

The association between MRKH syndrome and pituitary macroadenoma or macroprolactinoma is extremely rare. To the best of our knowledge, prolactinsecreting pituitary macroadenoma has not been reported in patients with MRKH syndrome. In the present case, both Mullerian agenesis and macroprolactinoma coexisted along with panhypopituitarism secondary to pituitary macroadenoma. There has been no definite explanation for the coexistence of these two anomalies in this patient as the number of similar cases are limited. To date, there has been only two reported cases of the association of MRKH syndrome with pituitary adenoma. Both cases reported patients with MRKH syndrome who presented with non-functioning pituitary microadenoma and normal secondary sexual characteristics $(8,9)$. Both cases also showed normal pituitary hormonal axes. The most recent case reported a patient with MRKH syndrome who presented with pituitary stalk interruption syndrome (10). Pituitary stalk interruption syndrome is a congenital disorder characterised by the triad of an absent or exceedingly thin pituitary stalk, an ectopic or absent posterior pituitary and/or absent or hypoplastic anterior pituitary.

It is noteworthy to mention that the diagnosis of MRKH syndrome imposes a significant psychological burden on patients because of the associated infertility. Surgically assisted reproductive techniques including uterine transplant and surrogacy may be viable options with regard to fertility (6), in the presence of intact hypothalamic-pituitary-gonadotrophic axes. However, the additional presence of abnormal pituitary hormones as in this patient, particularly panhypopituitarism, would impose even greater impact not only psychologically but also metabolically leading to cardiovascular, morbidity and mortality risks.

\section{Declaration of interest}

The authors declare that there is no conflict of interest that could be perceived as prejudicing the impartiality of the research reported.

\section{Funding}

This study did not receive any specific grant from any funding agency in the public, commercial or not-for-profit sector.

\section{Patient consent}

Written informed consent has been obtained.

\section{Author contribution statement}

Nur Aisyah Zainordin was the treating physician during the patient visit. She prepared the initial draft of the article, co-ordinated with co-authors, and prepared the final manuscript. Co-author Fatimah Zaherah was the consultant responsible for overseeing the case and the discussion of manuscript review. Co-author Aini Eddy, Sharifah Faradila and Aimi Abdullah were involved in the manuscript review and discussion. Rohana Abdul Ghani is a senior consultant endocrinologist who provided clinical input for the patient management and responsible for manuscript discussion and final review.

\section{References}

1 Pizzo A, Laganà AS, Sturlese E, Retto G, Retto A, De Dominici R \& Puzzolo D. Mayer-rokitansky-kuster-hauser syndrome: embryology, genetics and clinical and surgical treatment. ISRN Obstetrics and Gynecology 20132013 628717. (https://doi. org/10.1155/2013/628717)

2 Ledig S \& Wieacker P. Clinical and genetic aspects of MayerRokitansky-Küster-Hauser syndrome. Medizinische Genetik 201830 3-11. (https://doi.org/10.1007/s11825-018-0173-7)

3 Herlin MK, Petersen MB \& Brännström M. Mayer-Rokitansky-KüsterHauser (MRKH) syndrome: a comprehensive update. Orphanet Journal of Rare Diseases 202015 214. (https://doi.org/10.1186/s13023-02001491-9)

4 Yatavelli RKR \& Bhusal K. Prolactinoma. In StatPearls. Treasure Island (FL): StatPearls Publishing LLC, 2021.

5 De Menis E, Visentin A, Billeci D, Tramontin P, Agostini S, Marton E \& Conte N. Pituitary adenomas in childhood and adolescence. Clinical analysis of 10 cases. Journal of Endocrinological Investigation 200124 92-97. (https://doi.org/10.1007/BF03343820)

6 Committee on Adolescent Health Care. ACOG Committee Opinion No. 728: Mullerian agenesis: diagnosis, management, and treatment. Obstetrics and Gynecology 2018131 e35-e42. (https://doi.org/10.1097/ AOG.0000000000002458) 
7 Herlin M, Bjørn AM, Rasmussen M, Trolle B \& Petersen MB. Prevalence and patient characteristics of Mayer-Rokitansky-Küster-Hauser syndrome: a nationwide registry-based study. Human Reproduction 201631 2384-2390. (https://doi.org/10.1093/humrep/dew220)

8 Eom KS, Kim BR, Kim JM \& Kim TY. Coexistence of pituitary macroadenoma and Mayer-Rokitansky-Küster-Hauser syndrome. Journal of Clinical Neuroscience 2009 16 719-720. (https://doi. org/10.1016/j.jocn.2008.06.019)
9 Kannan RB, Nayak A, Varadhan L, Varughese G \& Jose B. MayerRokitansky-Kuster-Hauser syndrome and pituitary adenoma: a co-incidence? Endocrine Abstracts 201434 P143. (https://doi. org/10.1530/endoabs.34.P143).

10 MaWanlu,Xi Wang,Mao Jiangfeng et al.A Rare Case of Coexistence of Pituitary Stalk Interruption Syndrome and Mayer-Rokitansky-KüsterHauser Syndrome.Research Square2020(https://doi.org/10.21203/ rs.3.rs-91618/v1)

Received in final form 7 November 2021

Accepted 16 November 2021 